\title{
Age-related macular degeneration and the aging eye
}

\author{
Rita Ehrlich' \\ Alon Harris' \\ Nisha S Kheradiya' \\ Diana MWinston' \\ Thomas A Ciulla ${ }^{3}$ \\ Barbara Wirostko² \\ 'Indiana University School of \\ Medicine, Department \\ of Ophthalmology, Indianapolis, \\ IN, USA; ${ }^{2}$ Stony Brook University \\ Medical Center Research Way, \\ Department of Ophthalmology, Stony \\ Brook, NY, USA; ${ }^{3}$ Retina Service, \\ Midwest Eye Institute, Attending \\ Physician and Surgeon, Methodist \\ Hospital, Indianapolis, IN, USA
}

Correspondence: Alon Harris Department of Ophthalmology, Indiana University School of Medicine, 702 Rotary Circle, Indianapolis, IN 46202, USA

Tel +I 3172780177

Fax + I 3172781007

Email alharris@indiana.edu

\begin{abstract}
Age-related macular degeneration (AMD) is an ocular disease that causes damage to the retinal macula, mostly in the elderly. Normal aging processes can lead to structural and blood flow changes that can predispose patients to AMD, although advanced age does not inevitably cause AMD. In this review, we describe changes that occur in the macular structure, such as the retinal pigment epithelium and Bruch's membrane, with advancing age and in AMD. The role of genetics in AMD and age-related changes in ocular blood flow that may play a role in the pathogenesis of AMD are also discussed. Understanding the pathophysiology of AMD development can help guide future research to further comprehend this disease and to develop better treatments to prevent its irreversible central vision loss in the elderly.
\end{abstract}

Keywords: age-related macular degeneration, aging, blood flow, eye, retina, macula, vision

\section{Introduction}

Age-related macular degeneration (AMD) is an ocular disease that involves the posterior aspect of the retina called the macula. The macula facilitates central vision and permits high-resolution visual acuity due to its dense concentration of cone photoreceptors (Fine et al 2000). There is an early and late stage of AMD. In the early stage, there is formation of large drusen and pigmentary abnormalities (Fine et al 2000). The late stage is divided into two groups: nonexudative or "dry" form and an exudative/neovascular or "wet" form. The nonexudative form is characterized by atrophic changes in the macula and clinically, has a slower deterioration and better preservation of visual acuity than exudative AMD (Fine et al 2000). Exudative AMD involves choroidal neovascularization, which is the formation of new abnormal blood vessels in the choriocapillaries through Bruch's membrane (Figure 1). These vessels have a greater tendency of leakage and bleeding into the macula, ultimately leading to irreversible damage to the photoreceptors if left untreated (Fine et al 2000). Hence, the exudative form accounts for most cases of significant visual loss from AMD (Ciulla et al 2001).

AMD is the leading cause of visual deterioration and legal blindness in patients over 60 years of age (Klein et al 1997). The loss of central vision and high-resolution visual acuity from untreated AMD can lead to an irreversible loss of reading, facial recognition, and driving (Fine et al 2000). Changes in the macula occur over time and occur more often in the elderly. The prevalence of AMD among 40- to 49 -year-olds is $2.1 \%$, which increases dramatically to $35 \%$ among those over 80 years of age (National Eye Institute 2007). Additionally, the disease can progress with approximately $10 \%-20 \%$ of patients with nonexudative AMD progressing to the exudative form if untreated (Hyman and Neborsky 2002). Furthermore, almost 40\% of patients have bilateral disease (Topouzis et al 2006). The risk factors for AMD include ethnicity, gender, hypertension, genetics, diet, and sunlight exposure. Studies 


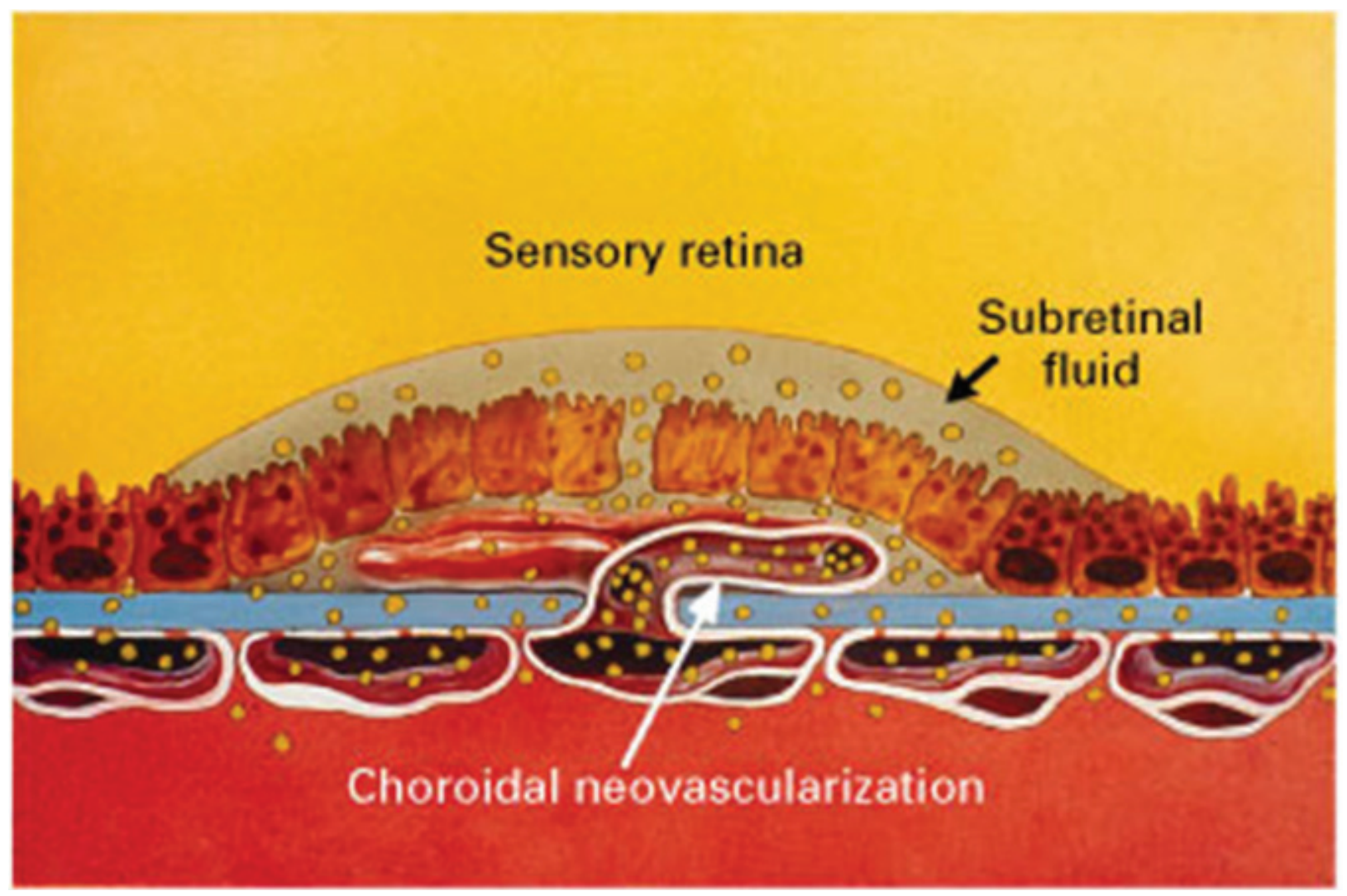

Figure I An illustration of the late form of AMD, showing the growth of the choroidal neovascularization from the choroicapillaris through the Bruch's membrane into the subretinal space. These new vessels are abnormal and have a higher tendency of leakage and bleeding. Reprinted with permission from Bressler NM, Bressler SB, Fine SL 1988. Age-related macular degeneration. Surv Ophthalmol, 32:375-413. @ 1998 Elsevier.

have shown that the late stages of AMD are more common in whites than in other ethnic groups and that women are affected more than men (Hyman and Neborsky 2002). Nevertheless, the strongest, most consistent risk factors are smoking and age (Hyman and Neborsky 2002).

Although aging will not inevitably lead to AMD development, there are changes with age that can predispose the eye to development of AMD. Primary senescence of retinal pigment epithelial cells and Bruch's membrane can lead to accumulation of metabolic debris and drusen. Furthermore, primary abnormalities in ocular perfusion worsen with age, secondarily causing dysfunction of the retinal pigment epithelial cells, predisposing eyes to AMD. These anatomical changes together with an individual's genetic make-up and environmental risk factors set the stage for the development of AMD (Ciulla et al 2001).

In this report, we focus on the role that aging plays in the pathogenesis of AMD. We start from a structural approach with alterations in the retinal pigment epithelium-Bruch's membrane-choriocapillaris complex. Then we focus on the vascular changes with an attention to ocular blood flow alterations that correlate with AMD lesion development. Finally, we discuss the role of genetics in development of AMD.

\section{Structural changes with age and in AMD}

The outer retina is made up of the retinal pigment epithelium and photoreceptors. The retinal pigment epithelial cells form the outer blood-retinal barrier to facilitate selective transport between the choroidal blood vessels and the outer retina (Salvi et al 2006). Therefore, they play a major role in rod and cone photoreceptor integrity. The retinal pigment epithelium is a phagocytic system that is essential to the renewal of photoreceptors (de Jong 2006). When the tips (or outer segments) of photoreceptors are shed, retinal pigment epithelial cells have been shown to engulf and to degrade its components (de Jong 2006). Burns and FeeneyBurns (1980) speculate that the retinal pigment epithelium then transports the engulfed cytoplasmic material through Bruch's membrane into the choriocapillaries.

Age-related processes that occur in the retinal pigment epithelium-Bruch's membrane-choriocapillaris complex 
can precede the development of AMD (Liang and Godley 2003). With advancing age, retinal pigment epithelial cells undergo an increase in pleomorphism, a decrease in the concentration of cells in the posterior pole, and decreased melanin content (Salvi et al 2006). Senescent retinal pigment epithelial cells accumulate metabolic debris from remnants of incomplete degradation of phagocytized rod and cone membranes (Ciulla et al 2001). Fatty acids from the outer segment receptors are degraded by lipid peroxidation, a process that increases with age in the macula (Zarbin 2004). Susuki et al (2007) found oxidized phospholipids present in photoreceptors and retinal pigment epithelial cells in the macula of the normal eyes, with increased quantities with advanced age. Metabolic debris includes lipofuscin, an undegradable byproduct of outer segment photoreceptor metabolism, which increases in concentration within retinal pigment epithelial cell cytoplasm over time (Feeney-Burns et al 1984). It is believed that lipofuscin and fatty acids are continuously exposed to light and high oxygen tension, which initiates the production of reactive oxygen species (Zarbin 2004). It is further postulated that these cause oxidative damage to the cellular mitochondria and to mitochondrial DNA, which is most susceptible to this oxidative stress (Liang and Godley 2003). Injury to the mitochondria leads to a reduction in energy production, compromises cellular and physiologic functioning, and can subsequently signal apoptosis in retinal pigment epithelial cells (Liang and Godley 2003; Zarbin 2004). Increased apoptosis eventually causes a decreased number of retinal pigment epithelial cells (Cai et al 1999). In order to counteract the formation of reactive oxygen species, retinal pigment epithelial cells contain anti-oxidants, which decrease with age (Liang and Godley 2003). The Age-Related Eye Disease Study (AREDS) has demonstrated that the administration of anti-oxidants, such as vitamins B, C, E, and zinc, significantly slows the rate of progression from high-risk nonexudative AMD to exudative AMD (AREDS 2001).

Retinal pigment epithelial cell damage also leads to the production of an abnormal extracellular matrix, which alters retinal pigment epithelium function, and causes choriocapillary loss associated with geographic atrophy (Zarbin 2004). Damaged retinal pigment epithelial cells can promote an inflammatory response that has been implicated in the development of AMD and the development of neovascularization (Moshfeghi and Blumenkranz 2007). Once damaged, these retinal pigment epithelial cells can secrete growth factors, such as vascular endothelial growth factor (VEGF), basic fibroblast growth factor (bFGF), and transforming growth factor beta (TGF- $\beta$ ). These cytokines play a major role in the formation of choroidal neovascularization in AMD (Lutty et al 1999). Recent evidence suggests that within the eye, there is a fine balance between endogenous angiogenic and anti-angiogenic growth factors. Imbalances between these factors can lead to choroidal neovascularization (Bhutto et al 2006). VEGF has been found to be one of the key angiogenic growth factors. The family of VEGF proteins, including VEGF-A, VEGF-B, VEGF-C, VEGF-D, and PlGF, exert their function through tyrosine kinase receptors (Andreoli and Miller 2007). VEGF has been shown to be present in choroidal neovascularization membranes (Otani et al 2002). Conversely, pigment epithelium derived factor (PEDF) is a potent endogenous anti-angiogenic growth factor. PEDF has been shown to significantly suppress VEGF-induced proliferation and migration of vascular endothelial cells (Bhutto et al 2006). Decreased PEDF levels have been reported in the vitreous samples of exudative AMD patients (Holekamp et al 2002). Choroidal PEDF immunoreactivity is also reduced in AMD patients compared to age-matched controls (Bhutto et al 2006). Currently, medications targeting VEGF, such as bevacizumab and ranibizumab, are widely used in the treatment of neovascular AMD. Future therapies may involve anti-angiogenic growth factors, such as PEDF.

Various other proteins can also influence the pathogenesis of AMD. One such molecule, ubiquitin, is an intracellular protein that regulates numerous cellular processes, such as cell-cycle progression, signal transduction, transcriptional regulation, and endocytosis (Hershko and Ciechanover 1998). It has been implicated in immune responses and apoptosis (Hershko and Ciechanover 1998). Ubiquitin is prominent in ganglion cells, retinal pigment epithelium and age-related subretinal pigment epithelium deposits (Shang and Taylor 2004). The ubiquitin pathway involves two steps. The first phase is ubiquitination whereby a substance that needs to be degraded is tagged with specific amino acid sequences. Subsequently, tagged molecules undergo proteolytic degradation through ubiquitin processing enzymes. This process allows damaged or obsolete proteins to be degraded before they aggregate within the cytoplasm. Over time, this protective pathway becomes compromised (Shang and Taylor 2004). Additionally, hydrogen peroxide $\left(\mathrm{H}_{2} \mathrm{O}_{2}\right)$ increases the level of oxidized glutathione, which further interferes with ubiquitination (Shang and Taylor 2004). Ubiquitin, without the active ubiquitin processing enzymes, is found in subretinal pigment epithelial deposits (Shang and Taylor 2004). It is speculated that with age, ubiquitinated proteins in retinal pigment epithelial cells do not undergo degradation by 
the ubiquitin processing enzymes (Shang and Taylor 2004). Therefore, changes in the ubiquitin pathway compromise protein degradation and contribute to subretinal pigment epithelial protein accumulation, which occurs in both advanced age and AMD.

Drusen are subretinal pigment epithelium deposits that are the clinical hallmark of AMD (Sivaprasad et al 2005). There are two classifications of drusen, depending on where they are located: basal linear and basal laminar deposits. Basal linear deposits accumulate between the basal lamina of the retinal pigment epithelium and the inner collagenous zone of Bruch's membrane (Sivaprasad et al 2005). Basal laminar deposits are found between the basement membrane and plasma membrane of the retinal pigment epithelium and consist of basement membrane proteins and collagen. The thickness of drusen deposits have been shown to correlate with the degree of retinal pigment epithelium degeneration, photoreceptor fallout, and vision loss (Sarks et al 2007). Drusen size, number, and degree of confluence are significant risk factors for the development of AMD (Bressler et al 1990). Soft and confluent drusen are a classic clinical hallmark of AMD. Interestingly, the molecular composition of drusen and the atherosclerotic deposits within blood vessels share many similarities (Sivaprasad et al 2005). Both contain vitronectin (a multifunctional plasma and extracellular matrix protein), apolipoprotein E, complement components and lipids (Mullins et al 2000). Nevertheless, a proteonomic analysis comparing drusen in AMD patients and age-matched controls found that although two-thirds of the proteins were similar between the two groups, one-third of the proteins detected in drusen from AMD patients were not found in controls (Crabb et al 2002). Furthermore, it is postulated that the development of drusen is most likely a selective and active process than a passive accumulation of proteins and lipids (Crabb et al 2002). Therefore, drusen formation may be influenced by an individual's genetic predisposition or environmental stressors, which helps explain why all aged patients do not develop AMD (Figure 2).

With age, drusen can further accumulate on either side of the elastin layer in Bruch's membrane (Feeney-Burns and Ellersieck 1985). Bruch's membrane is a connective tissue layer that lies between the metabolically active retinal pigment epithelial cells and the choriocapillaris. It supplies nutrition to retinal pigment epithelial cells (Sivaprasad et al 2005) and regulates ionic and metabolic exchange between the retinal pigment epithelium and choriocapillaries (Ramrattan et al 1994). With age, Bruch's membrane increases in thickness from $2 \mu \mathrm{m}$ in the first decade of life to $4.7 \mu \mathrm{m}$ in the 10th decade (Ramrattan et al 1994). Thickened Bruch's membrane can predispose itself to crack formation (Ciulla et al 2001). In patients with exudative AMD, Bruch's membrane is more calcified and fragmented than in age-matched controls or patients with nonexudative AMD. These cracks can facilitate the development of choroidal neovascular membranes thus transforming nonexudative to exudative AMD (Spraul and Grossniklaus 1997).

The increased thickness of Bruch's membrane involves both the elastin and collagenous layers and results from decreased degradation and increased production of the extracellular matrix (Zarbin 2004). Matrix mellatoproteinases (MMPs), which play a role in the degradation of extracellular matrix, and the tissue inhibitors of metalloproteinase (TIMPs), which regulate the activity of MMPs, have been found to change with age (Zarbin 2004). Increased content of the inactive form of MMP-2, MMP-9 as well as TIMP-3 have been found in the Bruch's membrane of aged patients (Zarbin 2004). Additionally, MMPs can facilitate choroidal neovascularization. Activated by VEGF, MMPs break down the extracellular matrix, allowing the growth of new vessels (Steen et al 1998). MMP-2 and MMP-9 have been localized to areas of new vessel development and to the enveloping Bruch's-like membrane suggesting that these enzymes may be cooperatively involved in the progressive growth of choroidal neovascular membranes in AMD. Nevertheless, the amounts of noncollagen proteins, lipid deposits, heparin sulfate, laminin, and fibronectin are also increased in Bruch's membrane with age (Zarbin 2004). Additionally, glycosaminoglycans increase in size and advanced glycation end products accumulate within Bruch's membrane over time (Zarbin 2004). The accumulation of cholesterol esters in Bruch's membrane with advancing age is similar to that observed in the arterial intima in atherosclerosis (Curcio et al 2001). This increase in lipid content creates a hydrophobic environment, which changes the flux of solutes and fluids across Bruch's membrane (Bird and Marshall 1986). Therefore, changes in thickness, molecular composition and electric charge leads to an age-related loss of permeability in Bruch's membrane and alters the movement of nutritional factors from the choriocapillaries toward the retinal pigment epithelial layer and subretinal space, thereby affecting the available nutrients for the outer retina (Zarbin 2004).

\section{Ocular blood flow changes with age}

There is a progressive decrease in the thickness of the choroid from $193 \mu \mathrm{m}$ in the first decade of life to $84 \mu \mathrm{m}$ in the 10th decade (Ramrattan et al 1994). There is also a 


\section{Vascular Changes with Age Structural Changes with Age}

\author{
- Decreased thickness of \\ choroid
}

- Decrease in choriocapillary density

- Decreased lumen diameter of choriocapillaries

-Increased resistance to flow

-Reduced choroidal flow

- Reduced choroidal perfusion
-Senescence of RPE cells

-Accumulation of waste products and formation of drusen

-Thickening of Bruch's membrane

-Change in the composition of Bruch's membrane

- Changes in the extracellular matrix

-Increase in oxidative stress

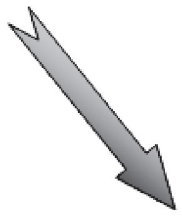

Genetic Predisposition and Environmental Risk Factors

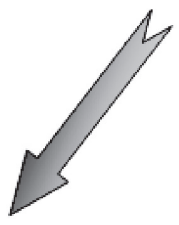

\section{AMD}

Figure $\mathbf{2}$ The vascular and nonvascular theory of AMD. The vascular theory, suggested by Friedman (1997), states that with increased resistance in the choroidal vessels, there is an increase in the osmotic gradient against which the retinal pigment epithelial cells must pump. This leads to retinal pigment epithelial cell dysfunction and the accumulation of metabolic debris and drusen formation. Furthermore, the decrease in choroidal perfusion causes ischemic/hypoxic injury and increased oxidative stress, which can signal choroidal neovascularization formation. On the other hand, in the nonvascular theory, senescent retinal pigment epithelial cells can lead to retinal pigment epithelial cell dysfunction, which in turn can cause choriocapillary atrophy. Over time, and individual's genetic predisposition and environmental stressors in the setting of structural and vascular changes make the eye more susceptible to AMD development.

$45 \%$ decrease in choriocapillary density and a $34 \%$ decrease in the lumen diameter of the choriocapillaries with advancing age (Ramrattan et al 1994). Reduced numbers of macular arterioles and decreased intensity of dye in the macular region have been demonstrated over time using indocyanine green angiography (Nishiyama et al 2001). These age-related changes were noted only in the arteries, but not in veins. Furthermore, choroidal blood flow changes have been reported with advancing age (Ramrattan et al 1994). Pulsatile ocular blood flow reflects the total pulsatile component of ocular blood flow and since most of the ocular blood volume is present in the choroid, it mainly represents the choroidal circulation (Sandhu et al 2007). Ravalico et al (1996) and Lam et al (2003) both found a decline in the pulsatile ocular blood flow with age. Animal models have shown a 35\%-42\% reduction in ocular blood flow and a 57\%-93\% increase in ocular vascular resistance in aged rats compared with juvenile or adult rats (Salter et al 1998). Age-related changes in the reflexive choroidal vasodilatation following hypoperfusion have also been shown in rat models (Fitzgerald et al 2005). Neurogenic choroidal blood flow control declines with age both in human and in animal studies (Fitzgerald et al 2005). With the use of laser Doppler flowmetry, Grunwald et al (1998) reported age-related decreases in choroidal blood flow in the fovea, which was related to decreases in blood volume but not velocity.

The use of color Doppler imaging provides measurements of the retrobulbar vasculature, namely the ophthalmic, central retinal and short posterior ciliary arteries. Retrobulbar changes have been demonstrated with advancing age. Age-related decreases in peak systolic velocity and end-diastolic velocity of the ophthalmic artery have been reported (Harris et al 2000; Lam et al 2003). Additionally, Pourcelot's resistive index was increased in the ophthalmic artery (Harris et al 2000, Lam et al 2003). Groh et al (1996) found a decrease in central retinal artery blood flow by 
approximately $6 \%-11 \%$ per decade with an increase in the resistive index. On the other hand, Harris et al (2000) found that central retinal artery flow velocity was unaffected by age in both sexes. Nevertheless, they did find that end-diastolic velocity of the posterior ciliary arteries decreased and the resistive index increased with age in women, but not in men.

The perfusion of the optic nerve head is also altered with increasing age. Boehm et al (2005) used laser Doppler flowmetry to find that volume and flow in the optic nerve decreased with age, suggesting that blood supply is reduced in elderly subjects. Neuroretinal rim and lamina cribrosa blood flow also decreases with advancing age (Embelton et al 2002). Additionally, Rizzo et al (1991) used a laser Doppler technique to demonstrate that advancing age is associated with a reduction in capillary red blood cell speed at the optic nerve head.

\section{Ocular blood flow changes in AMD}

Atherosclerosis has been postulated to be associated with late stages of AMD through its effect on the choroidal circulation and possible deposition of lipids in the Bruch's membrane (Freidman 2000; Curcio et al 2001). A history of cardiovascular disease and increased levels of cholesterol and stage 2 hypertension are strongly associated with neovascular AMD (Hogg et al 2008). The Rotterdam Eye Study demonstrated that persons with subclinical cardiovascular disease, as manifested by carotid artery plaques, were nearly five times more likely to have late AMD compared to patients without such plaques (Vingerling et al 1995a). The Multiethnic Study of Atherosclerosis (MESA) found an association between the severity of the intima-media thickness of the carotid artery and early AMD in black participants (Klein et al 2007a). Nevertheless, they did not find an association between severity of subclinical atherosclerosis and early AMD in the entire MESA cohort (Klein et al 2007a). The Women's Health Initiative Sight Exam Ancillary Study (WHISE) further reported a positive association between elevated systolic blood pressure and the presence of soft drusen, which correlated inversely with retinal pigment epithelial depigmentation and exudative AMD (Klein et al 2007b). Diastolic blood pressures and mean arterial blood pressures positively correlated with an increased risk of early AMD even after controlling for age (Klein et al 2007b). Although the relationship between blood pressure and AMD is not always consistent, several studies have demonstrated an association. In the Beaver Dam Eye Study, persons with hypertension at baseline were approximately two to three times more likely to develop neovascular macular degeneration after 10 years of follow-up than age-matched controls (Klein et al 2003). Furthermore, the Age-Related Macular Degeneration Risk Factor Study reported hypertension and high cholesterol to be associated with increased risk of neovascular AMD (Hyman et al 2000).

Since the choriocapillaris supplies the retinal pigment epithelial cells and the outer retina, a primary perfusion defect in the choriocapillaries could lead to loss or dysfunction of retinal pigment epithelial cells (Ciulla et al 2001). Spraul et al (1996) found increased total luminal area of peripheral choroidal vessels and decreased density of choroidal vessels in the macular area of eyes with AMD. In his theory on the development of AMD, Friedman (1997) hypothesized that with age, lipoid infiltration occurs within the sclera, Bruch's membrane and the wall of blood vessels, which leads to a decrease in the scleral and choroidal vessels compliance. This decrease in compliance can increase the resistance of the choroidal blood vessels and decrease choroidal perfusion (Friedman 1997). The elevated resistance can also increase intravascular pressure and raise hydrostatic pressure. Furthermore, scleral rigidity has been shown to increase with age (Lam et al 2003; Pallikaris et al 2005) and in patients with AMD (Friedman et al 1989). All of these changes in the choriocapillaris can impair the retinal pigment epithelial cell transport, promote increased debris accumulation and cause dysfunction of the retinal pigment epithelial cells (Friedman 1997) to set the stage for AMD development.

Blood flow analysis in patients with AMD shows decreased choroidal blood flow, velocity and volume in the fovea in AMD patients (Grunwald et al 2005). In addition, the severity of AMD increases in patients with the lowest circulatory parameters, possibly contributing to the ischemia and preceding the development of choroidal neovascularization (Grunwald et al 2005). With the use of the laser Doppler flowmetry, Metelitsina et al (2006) further investigated choroidal blood flow in the fovea of patients with early AMD with or without systemic hypertension. AMD patients with systemic hypertension had $16.7 \%$ lower blood flow compared to patients without hypertension (Metelitsina et al 2006). These results further suggest that systemic hypertension may contribute to the progression of AMD and the development of choroidal neovascularization (Metelitsina et al 2006). Sato et al (2006) also used the laser Doppler flowmetry to find that the pulsatility index, pulsatility ratio and resistive index were higher in patients with AMD and increased with disease severity. They found no difference between the mean blood velocity and flow rates and no differences in arterial diameter, suggesting that patients with AMD suffer from decreased 
compliance in the arterial vasculature (Sato et al 2006). Pulsatile ocular blood flow has been found to be decreased in exudative AMD compared to nonexudative AMD or normal subjects (Mori et al 2001). Chen et al (2001) found that the pulsatile ocular blood flow in eyes with drusen was lower than in eyes with choroidal neovascularization and higher in eyes with disciform scar, implying that hemodynamic changes can be partly responsible for asymmetry in some AMD patients. Nevertheless, a recent study of Caucasian patients by Sandhu et al (2007) demonstrated no differences between the pulsatile ocular blood flow in asymmetric eyes of patients with AMD. Moreover, delayed choroidal filling time has been found in patients with dry AMD (Ciulla et al 2002). Prolonged choroidal filling phase was observed in $26 \%$ of eyes with early AMD using fluorescein angiography and in $32 \%$ of eyes using indocyanine green (Pauleikoff et al 1999). This prolonged filling was also associated with confluent drusen and geographic atrophy in the fellow eye (Pauleikoff et al 1999). Presence of macular watershed zones was also correlated with AMD and one study found that choroidal neovascularization arose from the macular watershed zone in $91 \%$ of cases (Ross et al 1998).

Changes in the retrobulbar blood flow can provide a better understanding of the primary or secondary role of decreased blood flow in AMD. In an animal model, selective damage to retinal pigment epithelial cells caused degenerative changes to the choriocapillaris, suggesting that degeneration of choriocapillaries in AMD can be secondary to dysfunction of the retinal pigment epithelial cells (Henkind and Gartner 1983; Del Priore et al 1996). Therefore, secondary damage by retinal pigment epithelium is more likely to affect local blood vessels like the short posterior ciliary arteries and choriocapillaris than the ophthalmic or central retinal arteries. Changes in these vessels can support a more primary role of decrease in blood flow in the development of AMD, although a secondary autoregulatory response to a primary change elsewhere can not be ruled out (Ciulla et al 1999). Friedman et al (1995) reported that there is elevated pulsatility within all vessels including the ophthalmic, central retinal, and short posterior ciliary arteries, which can indicate an increase in the resistive index. Hosal et al (1998) reported that patients with AMD have lower blood velocity and higher resistive index in all retrobulbar vessels including the ophthalmic artery. Studies have found that blood flow velocity was lower in the ophthalmic artery and short posterior ciliary arteries of eyes with choroidal neovascularization compared with eyes without choroidal neovascularization (Uretmen et al 2003; Ciulla et al 1999). Various studies have reported decreases in the velocity of the short posterior ciliary arteries in AMD patients (Friedman et al 1995; Dimitrova et al 2002). Ciulla et al (1999) also found a decrease in the end-diastolic velocity and an increase in the resistive index of the central retinal artery. There are a couple of hypotheses for this change in the central retinal artery blood flow velocity. First, reductions in central retinal artery blood flow may be due to global decreases in retrobulbar blood flow from the ophthalmic artery and its branches. Secondly, decreased retinal blood flow may be related to retinal angiomatous proliferation. Yannuzzi et al (2001) described retinal angiomatous proliferation as a phenomenon which occurs in a subgroup of neovascular AMD patients, where angiomatous proliferation originates from the retina and extends posteriorly into the subretinal space, occasionally communicating with choroidal new vessels. Increased flow through new choroidal vessels could decrease flow in the retina and the central retinal artery. Nevertheless, further studies are necessary to determine whether primary or secondary vascular changes play a role in AMD.

Although the hemodynamic abnormalities do not directly indicate that the vascular abnormalities precede the development of AMD, they certainly suggest that hemodynamic changes play an important role in AMD pathogenesis. Friedman $(1997,2004)$ uses vascular theory to help explain what is known about the AMD pathophysiology. With advancing age, there is vascular stiffening and increased resistance. As overall vascular resistance increases, cerebral vascular resistance rises and leads to decreased choroidal perfusion and increased osmotic gradients against which retinal pigment epithelial cells must pump. This leads to accumulation of debris in the form of drusen. Furthermore, high choriocapillary pressure in the presence of calcification and fracture of Bruch's membrane and ischemic damage to retinal pigment epithelial cells can cause the secretion of vascular growth factors leading to the development of choroidal neovascularization (Friedman 1997, 2004). Although this theory has not been confirmed and does not elucidate the differences between races, it provides a well-rounded description of vascular alterations and how they contribute to the pathogenesis of AMD.

\section{Role of genetics in AMD}

Numerous population-based studies have reported differences in prevalence and incidence of AMD between various ethnic/racial groups (Klein et al 1999, 2006; Mitchell et al 1995; Vingerling et al 1995b). Several studies have demonstrated an increased incidence of 
AMD within families to provide further support for the role of genetics in AMD (Seddon et al 1997; Klaver et al 1998; Grizzard et al 2003). Therefore, it is important for investigators to continue to explore the role of genetics and environment on the development of AMD. Although genetic predisposition combined with environmental stressors seem to lead to disease development (Swaroop et al 2007), various studies have identified chromosomal abnormalities associated with AMD. Some of the chromosomal regions associated with AMD include 1q31-32, 6q21 and 10q26 (Gold et al 2006; Swaroop et al 2007). Within chromosome segment 10q26, specific gene regions include PLEKHA1, LOC387715 and HTRA1 (Swaroop et al 2007). Complement factor $H$, within chromosomal segment 1q31-32, is a key regulator in the alternative complement cascade and prevents uncontrolled complement activation (Yates et al 2007). Thakkinstian et al (2006) performed a meta-analysis between complement factor $\mathrm{H}$ $\mathrm{Y} 402 \mathrm{H}$ polymorphism and AMD to find that heterozygotes (TC) and homozygotes (CC) were, respectively, 2.5 and 6 times more likely to have AMD than homozygote patients with the TT genotype. Nevertheless, a Japanese study found that this complement factor $\mathrm{H}$ gene did not appear to increase risk of AMD within their population (Gotoh et al 2006). This appears to demonstrate that there are differences amongst various ethnic groups. Furthermore, within chromosome 6q21, polymorphisms of complement factor B and complement component 2 have been shown to be a risk predictor of AMD (Gold et al 2006). These correlations between complement-related genes and AMD further support the role of the inflammatory system in the development of AMD. In addition, a previous study by Mullins et al (2000) found that drusen can contain activated complement factors, acute phase reactants, coagulation proteins, immunoglobulins, and other proteins involved in the induction and activation of the immune response.

Not only can an individual's genetic predisposition influence the development of AMD, but it is also associated with the rate of disease progression. Seddon et al (2007) analyzed patients from the Age-Related Eye Disease Study (AREDS) to determine whether variations within the complement factor $\mathrm{H}$ gene and the LOC387715 gene have prognostic importance in AMD disease progression. Although genetic polymorphisms in the complement factor H Y402H and LOC387715 A69S were associated with progression to more advanced AMD, the LOC387715 genotype was also correlated with neovascular disease more than geographic atrophy. Patients who were homozygous for the risk alleles had a nearly $50 \%$ probability for AMD progression compared to the $5 \%$ risk in patients who are homozygous for the nonrisk alleles (Seddon et al 2007). Additionally, presence of the homozygous risk alleles combined with smoking and high body mass indices were associated with 19-fold increase of disease progression (Seddon et al 2007). Conversely, some genetic variations have been found to play a protective role in AMD development and progression. Apolipoprotein E is involved in transport and metabolism of lipid and cholesterol and in response to neural injury (Swaroop et al 2007). The $\varepsilon 4$ allele of apolipoprotein $\mathrm{E}$ has been associated with the reduced prevalence of AMD. There is strong evidence for the role of genetics in AMD, but further research is necessary to determine how genetic differences can ultimately lead to AMD.

\section{Conclusion}

Several systemic and ocular diseases increase in incidence and prevalence in the elderly population. Although AMD development shares many correlations with normal aging processes, other factors, such as genetic predisposition or environmental stressors, may account for disease development (Figure 2). Furthermore, the similarities between drusen and atherosclerosis demonstrate that a vascular component may play a role in AMD development. Apart from the structural changes, age-related alterations in ocular hemodynamics can precede and correlate with the AMD pathogenesis, which further supports the vascular theory of AMD.

Although aging alone is not sufficient to form AMD, it is apparent that the age-related changes and genetics are very important to the pathophysiology of AMD. Understanding the processes that develop with age and in age-related diseases can help guide new early treatment modalities. Further studies are warranted in order to learn how age-associated vascular and ocular structural changes lead to disease development. This will help generate the knowledge needed to identify target areas that may be useful to prevent disease progression through medical or surgical treatment.

\section{Acknowledgments}

Funded in part by an unrestricted grant from Research to Prevent Blindness, New York, NY. The authors would like to thank Lynne McCranor for her assistance with this manuscript.

\section{Disclosures}

None of the authors has any conflicts of interest to disclose.

\section{Reference}

Andreoli CM, Miller JW. 2007. Anti-vascular endothelial growth factor therapy for ocular neovascular disease. Curr Opin Ophthalmol, 18:502-8. 
Age-Related Eye Disease Study Research Group. 2001. A randomized, placebo-controlled, clinical trial of high-dose supplementation with vitamins $\mathrm{C}$ and $\mathrm{E}$, beta carotene, and zinc for age-related macular degeneration and vision loss. Arch Ophthalmol, 119:1417-36.

Bhutto IA, McLeod DS, Hasegawa T, et al. 2006. Pigment epithelium-derived factor (PEDF) and vascular endothelial growth factor (VEGF) in aged human choroid and eyes with age-related macular degeneration. Exp Eye Res, 82:99-110.

Bird JC, Marshall J. 1986. Retinal pigment epithelial detachments in the elderly. Trans Soc Ophthalmol UK, 105:674-82.

Boehm AG, Koeller AU, Pillunat LE. 2005. The effect of age on optic nerve head blood flow. Invest Ophthalmol Vis Sci, 46:1291-5.

Bressler NM, Bressler SB, Fine SL 1988. Age-related macular degeneration. Surv Ophthalmol, 32:375-413.

Bressler S, Maguire G, Bressler N, et al. 1990. Relationship of drusen and abnormalities of the retinal pigment epithelium to the prognosis of neovascular macular degeneration. Arch Ophthalmol, 108:1442-7.

Burns RP, Feeney-Burns L. 1980. Clinico-morphologic correlations of drusen of Bruch's membrane. Trans Am Ophthalmol Soc, 78:206-25.

Cai J, Wu M, Nelson KC, et al. 1999. Oxidant-induced apoptosis in cultured human retinal pigment epithelial cells. Invest Ophthalmol Vis Sci, 40:959-66.

Chen SJ, Cheng CY, Lee AF, et al. 2001. Pulsatile ocular blood flow in asymmetric exudative age related macular degeneration. $\mathrm{Br} J$ Ophthalmol, 85:411-15.

Ciulla TA, Harris A, Chung HS, et al. 1999. Color Doppler imaging discloses reduced ocular blood flow velocities in nonexudative age-related macular degeneration. Am J Ophthalmol, 128:75-80.

Ciulla TA, Harris A, Martin BJ. 2001. Ocular perfusion and age related macular degeneration. Acta Ophthalmol Scand, 79:108-15.

Ciulla TA, Harris A, Kagemann L, et al. 2002. Choroidal perfusion perturbations in non-neovascular age related macular degeneration. $\mathrm{Br} J$ Ophthalmol, 86:209-13.

Crabb JW, Miyagi M, Gu X, et al. 2002. Drusen proteome analysis: an approach to the etiology of age-related macular degeneration. Proc Natl Acad Sci USA, 99:14682-7.

Curcio CA, Millican CL, Bailey T, et al. 2001. Accumulation of cholesterol with age in human Bruch's membrane. Invest Ophthalmol Vis Sci, 42:265-74

de Jong PT. 2006. Age related macular degeneration. $N$ Engl J Med, 355:1474-85.

Del Priore LV, Kaplan HJ, Hornbeck R, et al. 1996. Retinal pigment epithelial debridement as a model for the pathogenesis and treatment of macular degeneration. Am J Ophthalmol, 122:629-43.

Dimitrova G, Tamaki Y, Kato S. 2002. Retrobulbar circulation in patients with age-related maculopathy. Eye, 16:580-6.

Embleton SJ, Hosking SL, Roff Hilton EJ, et al. 2002. Effect of senescence on ocular blood flow in the retina, neuroretinal rim and lamina cribrosa, using scanning laser Doppler flowmetry. Eye, 16:156-62.

Feeney-Burns L, Ellersieck MR. 1985. Age-related changes in the ultrastructure of Bruch's membrane. Am J Ophthalmol, 100:686-97.

Feeney-Burns L, Hilderbrand ES, Eldridge S. 1984. Aging human RPE: morphometric analysis of macular, equatorial and peripheral cells. Invest Ophthalmol Vis Sci, 25:195-200.

Fine SL, Berger JW, Maguire MG, et al. 2000. Age-related macular degeneration. $N$ Engl J Med, 342:483-92.

Fitzgerald MEC, Tolley E, Jackson B, et al. 2005. Anatomical and functional evidence for progressive age-related decline in parasympathetic control of choroidal blood flow in pigeons. Experimental Eye Research, 81:478-91.

Friedman E, Ivry M, Ebert E, et al. 1989. Increased scleral rigidity and agerelated macular degeneration. Ophthalmology, 96:104-8.

Friedman E, Krupsky S, Lane AM, et al. 1995. Ocular blood flow velocity in age-related macular degeneration. Ophthalmology, 102:640-6.

Friedman E. 1997. A hemodynamic model of the pathogenesis of age related macular degeneration. Am J Ophthalmol, 124:677-82.
Friedman E. 2000. The role of the atherosclerotic process in the pathogenesis of age-related macular degeneration. Am J Ophthalmol, 130:658-63.

Friedman E. 2004. Update of the vascular model of AMD. Br J Ophthalmol, $88: 161-3$.

Gold B, Merriam JE, Zernant J, et al. 2006. Variation in factor B (FB) and complement $2(\mathrm{C} 2)$ genes is associated with age related macular degeneration. Nat Genet, 38:458-62.

Gotoh N, Yamada R, Hiratani H, et al. 2006. No association between complement $\mathrm{H}$ gene polymorphism and exudative age related macular degeneration in Japanese. Hum Genet, 120:1-5.

Grizzard SW, Arnett D, Haag SL. 2003. Twin study of age related macular degeneration. Ophthalmic Epidemiol, 10:315-22.

Groh MJ, Michelson G, Langhans MJ, et al. 1996. Influence of age on retinal and optic nerve head blood circulation. Ophthalmology, 103:529-34.

Grunwald JE, Hariparasad SM, DuPont J, et al. 1998. Effect of aging on foveolar choroidal circulation. Arch Ophthalmol, 116:150-4.

Grunwald JE, Metelitsina TI, Dupont JC, et al. 2005. Reduced foveolar blood flow in eyes with increased AMD severity. Invest Ophthalmol Vis Sci, 46:1033-8.

Harris A, Harris M, Biller J, et al. 2000. Aging affects the retrobulbar circulation differently in women and men. Arch Ophthalmol, 118:1076-80.

Henkind P, Gartner S. 1983. The relationship between retinal pigment epithelium and the choriocapillaris. Trans Ophthalmol Soc UK, 103:444-7.

Hogg RE, Woodside JV, Gilchrist SE, et al. 2007. Cardiovascular disease and hypertension are strong risk factors for choroidal neovascularization. Ophthalmology, 115:1046-52.

Holekamp NM, Bouck N, Volpert O. 2002. Pigment epithelium-derived factor is deficient in the vitreous of patients with choroidal neovascularization due to age-related macular degeneration. Am J Ophthalmol, 134:220-7.

Hershko A, Ciechanover A. 1998. The ubiquitin system. Аnnu Rev Biochem, 67:425-79.

Hosal BM, Karakoç G, Gürsel E, et al. 1998. Color Doppler imaging of the retrobulbar circulation in age-related macular degeneration. Eur $J$ Ophthalmol, 8:234-8.

Hyman L, Schachat AP, He Q, Leske MC. 2000. Hypertension, cardiovascular disease, and age-related macular degeneration. Age-Related Macular Degeneration Risk Factors Study Group. Arch Ophthalmol, 118:351-8.

Hyman L, Neborsky R. 2002. Risk factors for age-related macular degeneration: an update. Curr Opin Ophthalmol, 13:171-5.

Klaver CC, Wolfs RC, Assink JJ, et al. 1998. Genetic risk of age related maculopathy. Population-based familial aggregation study. Arch Ophthalmol, 116:1646-51.

Klein R, Klein BE, Jensen SC, et al. 1997. The five years incidence and progression of age related maculopathy: the Beaver Dam Eye Study. Ophthalmology, 104:7-21.

Klein R, Klein BE, Cruickshanks KJ. 1999. The prevalence of age related maculopathy by geographic region and ethnicity. Prog Retin Eye Res, 18:371-89.

Klein R, Klein BE, Tomany SC, et al. 2003. The association of cardiovascular disease with the long-term incidence of age-related maculopathy: the Beaver Dam Eye Study. Ophthalmology, 110:273-80.

Klein R, Klein BE, Knudtson MD, et al. 2006. Prevalence of age related macular degeneration in $4 \mathrm{racial} / \mathrm{ethnic}$ groups in the multi-ethnic study of atherosclerosis. Ophthalmology, 113:373-80.

Klein R, Klein BE, Knudtson MD, et al. 2007a. Subclinical atherosclerotic cardiovascular disease and early age-related macular degeneration in a multiracial cohort: the Multiethnic Study of Atherosclerosis. Arch Ophthalmol, 125:534-43.

Klein R, Deng Y, Klein BE, et al. 2007b. Cardiovascular disease, its risk factors and treatment, and age-related macular degeneration: Women's Health Initiative Sight Exam ancillary study. Am J Ophthalmol, 143:473-83

Lam AKC, Chan ST, Chan H, et al. 2003. The effect of age on ocular blood supply determined by pulsatile ocular blood flow and color Doppler ultrasonography. Optom Vis Sci, 80:305-11. 
Liang FQ, Godley BF. 2003. Oxidative stress-induced mitochondrial DNA damage in human retinal pigment epithelial cells: a possible mechanism for RPE aging and age related macular degeneration. Experimental Eye Research, 76:397-403.

Lutty G, Grunwald J, Majji AB, et al. 1999. Changes in choriocapillaris and retinal pigment epithelium in age-related macular degeneration. Mol Vis, 5:35.

Metelitsina TI, Grunwald JE, DuPont JC, et al. 2006. Effect of systemic hypertension on foveolar choroidal blood flow in age related macular degeneration. Br J Ophthalmol, 90:342-6.

Mitchell P, Smith W, Attebo K, et al. 1995. Prevalence of age related maculopathy in Australia. The Blue Mountains Eye Study. Ophthalmology, 102:1450-60.

Mori F, Konno S, Hikichi T, et al. 2001. Pulsatile ocular blood flow study: decreases in exudative age related macular degeneration. $\mathrm{Br} J \mathrm{Oph}$ thalmol, 85:531-3.

Moshfeghi DM, Blumenkranz MS. 2007. Role of genetic factors and inflammation in age-related macular degeneration. Retina, 27:269-75.

Mullins RF, Russell SR, Anderson DH, et al. 2000. Drusen associated with aging and age-related macular degeneration contain proteins common to extracellular deposits associated with atherosclerosis, elastosis, amyloidosis, and dense deposit disease. FASEB J, 14:835-46.

National Eye Institute. 2007. Statistics and Data. Prevalence of Blindness Data. Data Tables [online]. Accessed Sept 18, 2007. URL: http://www. nei.nih.gov/eyedata/pbd_tables.asp.

Nishiyama Y, Mori K, Young-Duvall J, et al. 2001. Aging changes of the choroidal dye filling pattern in indocyanine green angiography of normal subjects. Retina, 21:237-42

Otani A, Takagi H, Oh H, et al. 2002. Vascular endothelial growth factor family and receptor expression in human choroidal neovascular membranes, Microvasc Res, 64:162-9.

Pallikaris IG, Kymionis GD, Ginis HS, et al. 2005. Ocular rigidity in living human eyes. Invest Ophthalmol Vis Sci, 46:409-14.

Patel N, Adewoyin T, Chong NV. 2007. Age related macular degeneration: a perspective on genetic studies. Eye, 11 May: doi:10.1038/ sj.eye. 6702844 .

Pauleikoff D, Spital G, Radermacher M, et al. 1999. A fluorescein and indocyanine green angiographic study of choriocapillaries in age related macular degeneration. Arch Ophthalmol, 117:1353-8.

Ramrattan RS, van der Schaft TL, Moony CM, et al. 1994. Morphometric analysis of Bruch's membrane, the choriocapillaris and chorid in aging. Invest Ophthalmol Vis Sci, 35:2857-64.

Ravalico G, Toffoli G, Pastori G, et al. 1996. Age related ocular blood flow changes. Invest Ophthalmol Vis Sci, 37:2645-50.

Rizzo JF, Feke GT, Goger DG, et al. 1991. Optic nerve head blood speed as a function of age in normal human subject. Invest Ophthalmol Vis Sci, 32:3263-72.

Ross R, Barofsky J, Cohen G, et al. 1998. Presumed macular choroidal watershed vascular filling, choroidal neovascularization, and systemic vascular disease in patients with age related macular degeneration. $\mathrm{Am}$ J Ophthalmol, 125:71-80.

Salter JM, Cassone VM, Wilkerson MK, et al. 1998. Ocular and regional cerebral blood flow in aging Fischer-344 rats. J Appl Physiol, 85:1024-9.

Salvi SM, Akhtar S, Currie Z. 2006. Ageing changes in the eye. Postgrad Med $J, 82: 581-7$.
Sandhu R, Sivaprasad S, Shah SP, et al. 2007. Pulsatile ocular blood flow in asymmetric age-related macular degeneration. Eye, 21:506-11.

Sato E, Feke GT, Menke MN, et al. 2006. Retinal haemodynamics in patients with age-related macular degeneration. Eye, 20:697-702.

Sarks S, Cherepanoff S, Killingsworth M, et al. 2007. Relationship of basal laminar deposit and membranous debris to the clinical presentation of early age-related macular degeneration. Invest Ophthalmol Vis Sci, 48:968-77.

Seddon JM, Ajani UA, Mitchell BD. 1997. Familial aggregation of age related maculopathy. Am J Ophthalmol, 123:199-206.

Seddon JM, Francis PJ, George S, et al. 2007. Association of CHF Y402H and LOC387715 A69S with progression of age related macular degeneration. JAMA, 297:1793-800.

Shang F, Taylor A. 2004. Function of the ubiquitin proteolytic pathway in the eye. Exp Eye Res, 78:1-14.

Sivaprasad S, Bailey TA, Chong VN. 2005. Bruch's membrane and the vascular intima: is there a common basis for age-related changes and disease? Clin Experiment Ophthalmol, 33:518-23.

Spraul CW, Lang GE, Grossniklaus HE. 1996. Morphometric analysis of the choroid, Bruch's membrane, and retinal pigment epithelium in eyes with age-related macular degeneration. Invest Ophthalmol Vis Sci, 37:2724-35.

Spraul CW, Grossniklaus HE. 1997. Characteristics of Drusen and Bruch's membrane in postmortem eyes with age-related macular degeneration. Arch Ophthalmol, 115:267-73.

Steen B, Sejersen S, Berglin L, et al. 1998. Matrix metalloproteinases and metalloproteinase inhibitors in choroidal neovascular membranes. Invest Ophthalmol Vis Sc, 39:2194-200.

Suzuki M, Kamei M, Itabe H, et al. 2007. Oxidized phospholipids in the macula increase with age and in eyes with age-related macular degeneration. Mol Vis, 13:772-8.

Swaroop A, Branham KE, Chen W, et al. 2007. Genetic susceptibility to age related macular degeneration: a paradigm for dissecting complex disease traits. Hum Mol Genet, 16:R174-82.

Thakkinstian A, Han P, McEvoy M, et al. 2006. Systematic review and meta analysis of the association between complement factor $\mathrm{H} \mathrm{Y} 402 \mathrm{H}$ polymorphism and age related macular degeneration. Hum Mol Genet, 15:2784-90

Topouzis F, Coleman AL, Harris A, et al. 2006. Prevalence of age-related macular degeneration in Greece: the Thessaloniki Eye Study. Am J Ophthalmol, 142:1076-9.

Uretmen O, Akkin C, Erakgün T, et al. 2003. Color Doppler imaging of choroidal circulation in patients with asymmetric age-related macular degeneration. Ophthalmologica, 217:137-42.

Vingerling JR, Dielemans I, Bots ML, et al. 1995a. Age-related macular degeneration is associated with atherosclerosis: The Rotterdam Study. Am J Epidemiol, 142:404-9.

Vingerling JR, Dielemans I, Hofman A, et al. 1995b. The prevalence of age related maculopathy in the Rotterdam Study. Ophthalmology, 102:205-10.

Yannuzzi LA, Negrão S, Iida T, et al. 2001. Retinal angiomatous proliferation in age-related macular degeneration. Retina, 21:416-34.

Yates JR, Sepp T, Matharu BK, et al. 2007. Complement C3 variant and the risk of age-related macular degeneration. $N$ Engl J Med, 357:553-61.

Zarbin MA. 2004. Current concepts in the pathogenesis of age related macular degeneration. Arch Ophthalmol, 122:598-614. 